\title{
Endoscopic Prudence to Assess Gastro-Esophageal Junction (GEJ); A Necessity Rather a Prerequisite Prior to Endoscopic Anti-Reflux Treatment in Gastro-Esophageal Reflux Disease (GERD) Patients
}

\section{Viswanath YKS*}

Professor of Surgery, Consultant Upper GI and Laparoscopic Surgeon, Surgical Directorate, Endoscopy Offices, James Cook University Hospital, Cleveland, UK *Corresponding Author: Viswanath YKS, Professor of Surgery, Consultant Upper GI and Laparoscopic Surgeon, Surgical Directorate, Endoscopy Offices, James Cook University Hospital, Cleveland, UK.

DOI: 10.31080/ASGIS.2020.03.0124
Received: January 31, 2020

Published: February 19, 2020

(c) All rights are reserved by Viswanath YKS., et al.

\section{Abstract}

Gastroesophageal reflux disease (GERD) is a common condition affecting more than $30 \%$ of the western adult population. Its impact on the quality of life is a well-known entity especially in patients with moderate to severe GERD. In the long term, it can lead to Barrett's metaplasia, dysplasia, and adenocarcinoma.

The 'triad of intrinsic lower esophageal sphincter (LES) function, extrinsic compression of crurae (pinch valve effect) and acute angle of HIS' are amongst a few main factors that contribute towards an effective anti-reflux barrier.

Recent advances in technology have enabled to treat GERD in indicated cases via an endoscope as a day case on outpatient basis. The effectiveness and efficacy of these endoscopy interventions are primarily determined by accurate pre-intervention assessment and selection of these patients.

Endoscopic fundoplication (EF) or Transoral incisionless fundoplication (TIF) and endoscopic radiofrequency (RF) anti-reflux therapy (STRETTA) are two well established day case procedures and evidence support that in selected cases, they impact positively on the improvement of efflux symptoms and dependency on PPI's1. The Stretta primarily effects via augmentation of intrinsic LES function, while EF via creating a flap valve. Both are known to be effective in patients with small hiatus hernia without pathological crural dilatation (CD) [1]. Currently, there is no endoscopy technique that can correct pathological crural dilation (extrinsic factor) and sliding hiatus hernia (more than $2.5 \mathrm{cms}$ ). Therefore, it is prudent to assess the GE junction (GEJ) and chose the right patient prior to offering an endoscopic treatment in a patient who is proven to have GERD. There is some evidence to support patient selection by means of an endoscopic gastro- esophageal junction flap valve (GEJFV) grading system [2]. The GEJ assessment comprises anatomical (endoscopy and imaging) and physiological (manometry) evaluation in conjunction with Ph studies. This article highlights the importance of endoscopic assessment and poses a few questions on selection criteria prior to endoscopic anti-reflux therapy. Keywords: Gastroesophageal Reflux Disease (GERD); Anti-reflux Therapy; Stretta

\section{Core Tip}

Assessment of gastroesophageal junction flap valve (GEJFP) before endoscopic antireflux therapy is important, particularly to distinguish from pathological CD and sliding HH. This recognition in conjunction with other factors such as the blunt angle of HIS and prolapsing gastric mucosa gives added information on accurate grading of GEJFV. One should make a note of the presence or absence of syncing of crural impression and Z line along with whether the $\mathrm{Z}$ line is visualized on scope retroversion. Validated new endoscopic scoring systems to assess GEJFP are needed to prognosticate more accurately outcomes after modern endoscopic anti-reflux treatments.

\section{Introduction}

The GERD is a common condition affecting $30 \%$ of the adult population and is one of the common chronic diseases in the western world. Its impact on the quality of life professionally, socially and personally is only an estimate, in reality, difficult to impel a true appraisal.

Not long ago, all fit and willing GERD patients with an impact on the quality of life, where standard medical and lifestyle measure failed, were offered laparoscopic fundoplication with crural repair [1-3]. This was the case whether the GERD was uncomplicated or complicated by severe esophagitis with or without a hiatus hernia.

Recent past endoscopic and technological advances have enabled attending endoscopist, to carry out complex therapeutic treatment procedures with relative ease. Ongoing research, advancing technology and added intelligent software are a few contributing factors. In the past two decades, lot many endoscopic anti-reflux technologies have been introduced, some without success [1]. Poor efficacy, costs, safety, and reproducibility are the main elements to account for their failure. 
Endoscopic fundoplication (EF) or Transoral Incisionless Fundoplication' (TIF) and Endoscopic RF treatments (Stretta) have stood the test of time in the last decade [1]. For an attending therapeutic endoscopist, to learn, incorporate the technique and reproduce the published results, vigorous patient selection is vital, this aside training and mentorship. A sub-selection of GERD patients not complicated by a large hiatus hernia, stricture and ulcer disease is para-amount to the successful outcome.

It is well documented, outcomes are better in GERD patients with a no or small hiatus hernia [HH (less than $2.0 \mathrm{cms}$ )], less than $2.0 \mathrm{~cm}$ crural dilatation (CD), absence of ulcerative esophagitis or a stricture. A good number of received referrals in our practice often, the crural dilatation [CD (the maximum distance between medial edges of the crurae)] is reported as hiatus hernia. It is vital to attempt to differentiate the two, as both can co-exist or could be present in isolation. Majority of normal subjects, crural impression (CI) syncs with the GE junction (Z line) and an important factor contributing to a competent anti-reflux barrier. Out of synchronization of $\mathrm{CI}$ with $\mathrm{Z}$ line with the especially associated proximal displacement of it needs careful assessment of GO junction on retroversion of the endoscope (Figure 1). One has to look at the displacement of gastric folds via the hiatus partly narrowing the space around the scope. Endoscopic assessment of GO junction is operator dependent and findings can become skewed especially patients who are restless and retch.

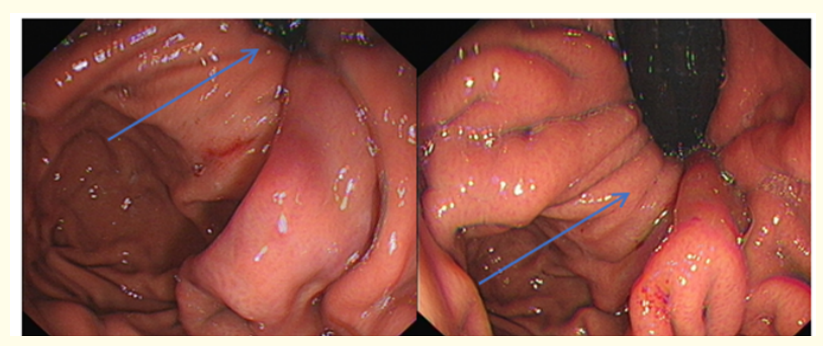

Figure 1: Prolapsing fundic mucosa annihilating the space around the scope, non-visualisation of $\mathrm{Z}$ line with indistinct crural impression (arrow).

For a patient with pathological CD (more than $2 \mathrm{cms}$ ) even without hiatus hernia, any endoscopic procedure is associated with less good outcomes. ONLY surgery in symptomatic and selected cases can address reflux problems via a crural repair with a fundoplication since crurae are extra-gastric structures. The crural repair becomes vital should the weakening of the antireflux barrier is mainly due to dilated hiatus. Currently, the forthcoming and established endoscopy antireflux therapies accentuate the LES pressure and enable the reduction of small hiatus hernias (less than $2.0 \mathrm{cms}$ ), without correcting the crural dilatation (CD).

Hills grading, a useful tool to grade 1 - 4 the GEJFV and well known to associate with increasing GERD symptoms and esophagitis at higher grades [4]. Also, assessing the prolapsing stomach, blunt angle of HIS, trueness of syncing of Z line with CI, could further assist the treating endoscopist to prognosticate the outcome (Figure 2 and 3). There is some evidence to support the existence of pathological crural dilatation [(CD) $>2.0 \mathrm{cms}$ ) and the presence of hiatus hernia ( $>2.0 \mathrm{cms}$ ), their meagre impact after treatment. This is besides $\mathrm{Ph}$ studies, manometry, impedance studies, and other assessments.

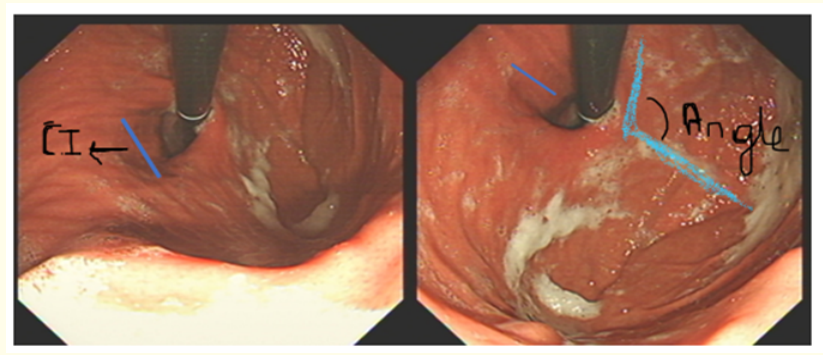

Figure 2: Crural Insufficiency (CI), obliteration of angle of HIS and sliding hiatus hernia (2 - $3 \mathrm{cms}$ ) Type 4.

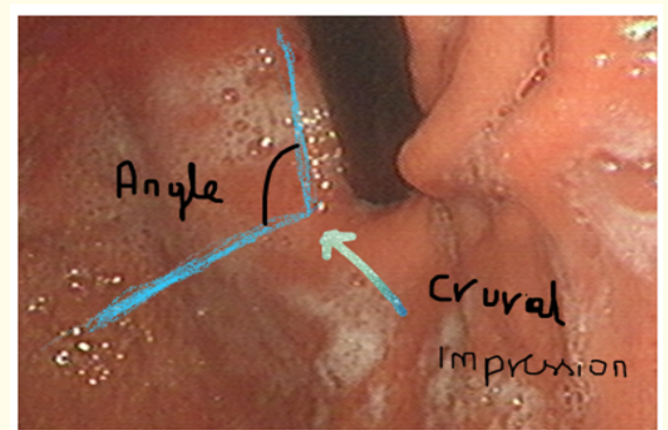

Figure 3: Flap valve not well formed (Type 2), angle of HIS blunt, Z line syncs with crural impression.

Whether a combination of endoscopic therapies further enhances the outcome in indicated GORD patients is unclear. Further auxiliary and critical studies are needed to corroborate the association of CD with or without HH. Moreover, other GO junctional structural changes such as; blunt angle of HIS, non-syncing of GO junction with $\mathrm{Z}$ line and presence of prolapsing of the stomach on the outcomes after endoscopic intervention needs new studies. We are looking at these factors in all patients undergoing endoscopic antireflux radiofrequency (Stretta) therapy in the unit.

\section{Evidence}

Handsdotter., et al. [3] studies 334 subjects (123 with reflux symptoms and 211without GERD), where they concluded Hills grading is reproducible and was superior to associate with reflux than an endoscopic assessment of the axial length of a hiatal hernia [2]. However, this study, could not verify it as a superior predictor. In a cross-sectional prospective study by Quach., et al. [2] involving 331 patients, showed that abnormal gastroesophageal valve was associated with high reflux score. They looked at both non-erosive reflux disease and reflux esophagitis. 
In 2018, Jeon., et al. [5] concluded the GE flap valve grading was well correlated with CT scan images. They also noted a positive association with the increasing angle of HIS on CT with the increase in the endoscopic grading of the valve. (It is evident from this study, that, angle of HIS escalation is coupled with more symptoms of GERD and higher grading of the GOJ flap valve. This raises the question, whether routine assessment and documentation of angle of HIS in addition to flap valve grading, adds further to prognosticate outcomes of endoscopic antireflux therapy or otherwise.

Chang., et al. [4]. examiner 36 children with GERD symptoms, concluded abnormal GOJ (flap valve) dysfunction is associated with more severe esophagitis. This study again corroborated the importance of a flap valve in the paediatric age group.

Oberg., et al. [6] have shown that grade $4 \mathrm{GEJFV}$ is associated with increased acid exposure in $75 \%$ of GERD patients. The prevalence of a defective LES, pathological acid reflux, mucosal inflammation and Barrett's metaplasia surge with greater grading of GEJFV.

\section{Conclusion}

All these studies highlight the merit of GEJFV grading in conjunction with GERD symptoms and associated pathology. Moreover, no well-validated studies are done to study its impact on outcomes after endoscopic antireflux therapies. Modification of 'Hills GEJFV grading, taking into consideration other factors such as the angle of HIS, syncing of Z line with CI and prolapsing of the stomach would further add value to choose the right patients before endoscopic antireflux therapy. This will enable attending interventional gastroenterologists to prognosticate outcomes after a proposed endoscopic antireflux therapy and signpost to appropriate endoscopic or surgical therapy when indicated.

\section{Bibliography}

1. Obuobi R., et al. "Trans-oral incisionless fundoplication vs stretta, two recognized endoluminal anti-reflux therapies; a systematic review and meta-analysis". International Surgery Journal 5.3 (2018): 765-772.

2. Duc T Quach., et al. "Abnormal Gastroesophageal Flap Valve Is Associated With High Gastresophageal Reflux Disease Questionnaire Score and the Severity of Gastroesophageal Reflux Disease in Vietnamese Patients With Upper Gastrointestinal Symptoms". Journal of Neurogastroenterology and Motility 24.2 (2018).

3. Hansdotter Ida., et al. "Assessment of the mechanical antireflux barrier at the gastroesophageal junction". Endoscopy International Open 04 (2016): E311-E317.

4. Chang K-C., et al. "Impacts of Endoscopic Gastroesophageal Flap Valve Grading on Pediatric Gastroesophageal Reflux Disease". PLoS One 9.9(2014): e107954.
5. Hye Kyung Jeon., et al. "Analysis of computed tomographic findings according to gastroesophageal flap valve grade". Korean Journal of International Medicine 33 (2018): 295-303.

6. Oberg., et al. "Endoscopic grading of the gastroesophageal valve in patients with symptoms of gastroesophageal reflux disease (GERD)". Surgical Endoscopy 13 (1999): 1184-1188.

\section{Assets from publication with us}

- Prompt Acknowledgement after receiving the article

- Thorough Double blinded peer review

- Rapid Publication

- Issue of Publication Certificate

- High visibility of your Published work

Website: www.actascientific.com Submit Article: www.actascientific.com/submission.php Email us: editor@actascientific.com

Contact us: +919182824667 